\title{
Multiple Particle Detection in a Neutron Monitor
}

\author{
Paul Evenson, ${ }^{a, *}$ John Clem, ${ }^{a}$ Pierre-Simon Mangeard, ${ }^{a}$ Waraporn Nuntiyakul, ${ }^{d}$ \\ David Ruffolo, ${ }^{b}$ Alejandro Sáiz, ${ }^{b}$ Achara Seripienlert $^{c}$ and Surujhdeo Seunarine ${ }^{e}$ \\ ${ }^{a}$ University of Delaware, \\ Newark DE, USA \\ ${ }^{b}$ Mahidol University, \\ Bangkok, Thailand \\ ${ }^{c}$ National Astronomical Research Institute of Thailand (NARIT), \\ Chiang Mai 50180, Thailand \\ ${ }^{d}$ Chiang Mai University, \\ Chiang Mai, Thailand \\ e University of Wisconsin River Falls, \\ River Falls WI, USA \\ E-mail: evenson@udel.edu, jmc@udel.edu, mangeard@udel.edu, \\ waraporn.n@cmu.ac.th, david.ruf@mahidol.ac.th, asaizrivera@gmail.com, \\ achara@narit.ac.th, surujhdeo.seunarine@uwrf.edu
}

Magnetic activity on the sun influences the flux of galactic cosmic rays at Earth in the process known as solar modulation. While most pronounced at $1 \mathrm{GeV}$ and below, it also operates at much higher energy, still exhibiting solar magnetic polarity dependence. Historically, an observational gap exists between approximately $17 \mathrm{GeV}$ (the highest geomagnetic cutoff) neutron monitor data and muon observations of primary cosmic rays that are mostly above $50 \mathrm{GeV}$. Detecting multiple neutrons from the same primary particle has recently been used to monitor the primary energy spectrum using data from a single neutron monitor. Over the past few years we have used details of the timing distribution from individual neutron detectors and pairwise correlations among adjacent detectors to improve the energy resolution of this technique. We present a further extension of our observations to pattern recognition of events comprising hits in multiple detectors in order to identify and study cases where more than one secondary particle from the same primary interacts in the neutron monitor. Our particular focus at present is to distinguish interactions of energetic hadrons from those generated by cores of small air showers.

$37^{\text {th }}$ International Cosmic Ray Conference (ICRC 2021)

July 12th - 23rd, 2021

Online - Berlin, Germany

\footnotetext{
*Presenter
} 


\section{Introduction}

When solar activity is high, the enhanced magnetic field in the solar wind reduces the cosmic ray flux at Earth in a process generally referred to as solar modulation. The overall magnetic polarity of the sun reverses at each solar maximum, resulting in an alternating pattern in the details of modulation in successive solar cycles. While most pronounced at $1 \mathrm{GeV}$ and below, modulation also operates at much higher energy, still exhibiting solar magnetic polarity dependence. The polarity dependence is observed to be in opposite directions at low and high energy [1-3]. The two mechanisms known to produce polarity dependence, gradient and curvature drift [4] and magnetic helicity [5] operate in opposite directions, suggesting that the drift mechanism dominates at low energy while helicity dominates at high energy.

Historically, an observational gap exists between approximately $17 \mathrm{GeV}$ neutron monitor data (the highest geomagnetic cutoff) and muon observations of primary cosmic rays that are mostly above $50 \mathrm{GeV}$. Our recent work has focused on techniques to extract spectral information directly from data takem at the Princess Sirindhorn Neutron Monitor on Doi Inthanon in Thailand (PSNM). The $17 \mathrm{GV}$ cutoff at PSNM allows observation of the high energy spectrum uncontaminated by the much more numerous low energy particles.

Detecting multiple neutrons from the same primary particle has long been discussed as a means to estimate the primary energy spectrum from a single neutron monitor [6]. The first definitive use was outlined in [7]. Over the past few years we have used details of the timing distribution of individual neutron detections to show that the shape of the cosmic ray spectrum at approximately $17 \mathrm{GV}$ is still being influenced by solar magnetic polarity [8]. Beginning in 2015 we upgraded the electronics at PSNM to record pairwise correlations among detectors to improve the energy resolution of this technique $[9,10]$. In this work we present a further extension of our observations to more complex combinations of detectors. Such multiple interactions show promise of better measurement of the energy of individual incident particles to give a more accurate estimate of the primary spectrum.

\section{Technical Details}

Figure 1 shows a photograph of PSNM, where 18 NM64 Units are enclosed in a polyethylene housing, generally called the reflector. Each Unit consists of a boron trifluoride proportional counter enclosed in a lead producer and polyethylene moderator. Additional moderated boron trifluoride counters, located outside the reflector, are referred to as bares.

We define the following terminology. We refer to each BF3 counter as a Unit. Cosmic rays incident on the atmosphere are referred to as primary particles. The products of interaction in the atmosphere are termed secondary particles, while a particle entering the monitor itself is called an incident particle. Thus, in the photo, a pencil beam of incident neutrons illuminates Unit 2. Signals recorded in the Units (most commonly a single neutron) are referred to as hits. Each hit is time-tagged and related to GPS time to an accuracy of 2 microseconds. Groups of hits are referred to as events, and events satisfying certain criteria are referred to as catches.

All hits are made available in real time to the software in the data acquisition system (DAQ). The hit rate in the NM64 Units is about $34 \mathrm{~Hz}$, while the bares operate at about $6 \mathrm{~Hz}$. The DAQ 


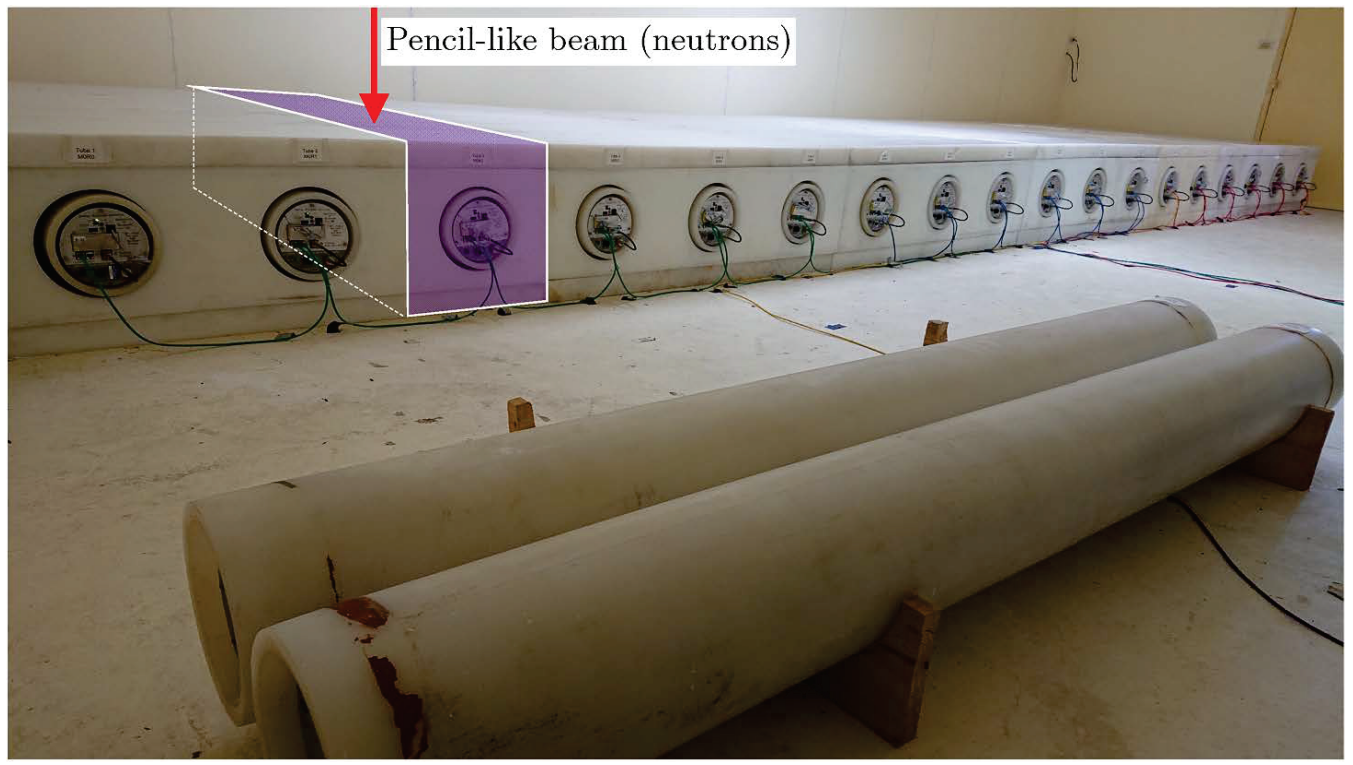

Figure 1: Annotated photograph of the Princess Sirindhorn Neutron Monitor (PSNM), indicating a simulated pencil-like beam of neutrons.

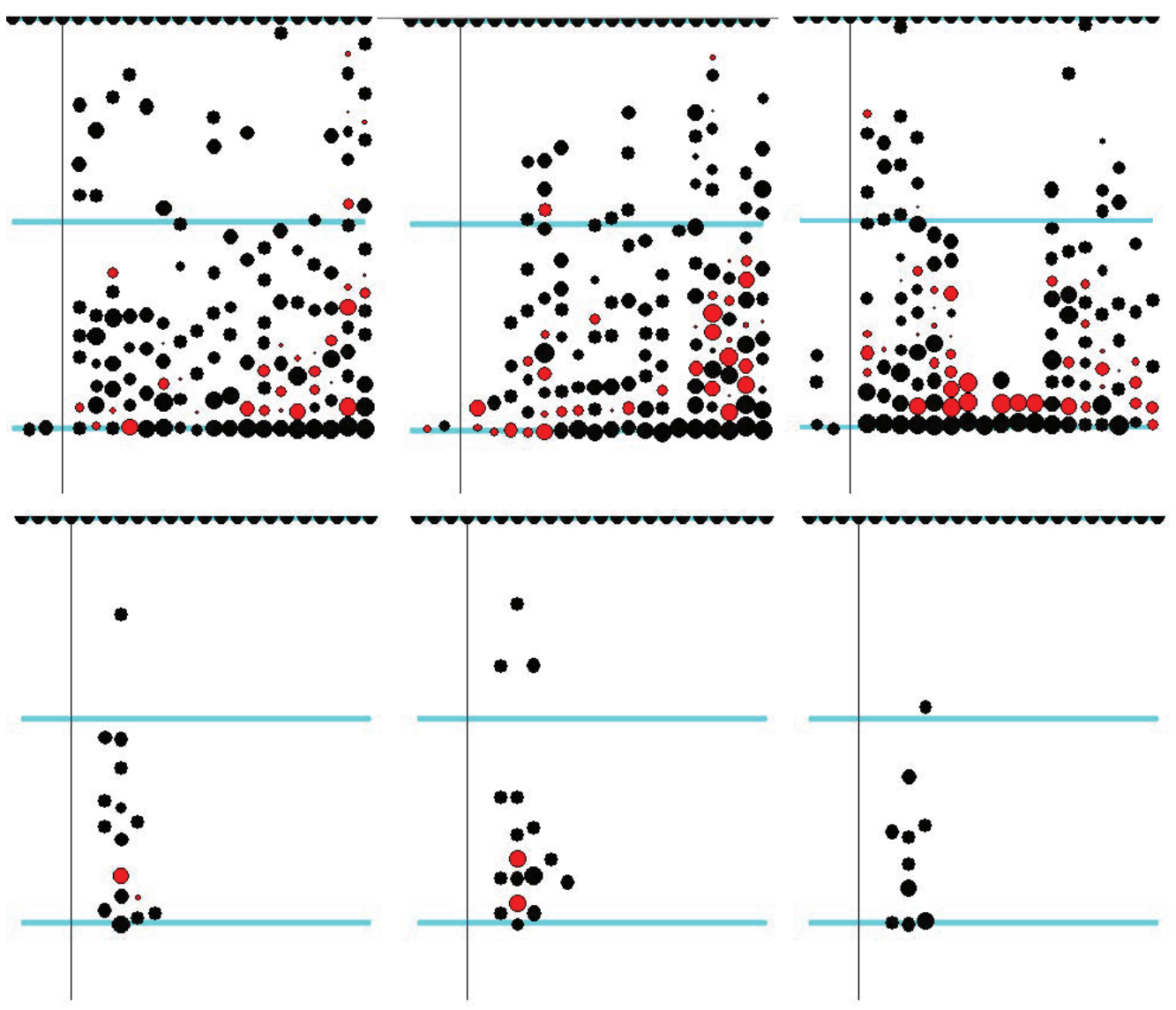

Figure 2: Top: Selected large events at PSNM. Bottom: Simulated $100 \mathrm{GeV}$ incident neutrons 
generates histograms of time delays between successive hits in individual Units, as well as pairwise delays in 54 different classes of Unit pairs (nearest neighbors, second nearest, etc.) More complex situations are recorded individually as catches, which form the basis for the analysis in this paper. Typically the only outputs are the histograms (hourly) and the catches, but it is possible to record all hits (the so-called HitSpool) for offline development of catch criteria.

Operation of the DAQ is illustrated in Figure 2. At the top are catches at PSNM resulting from dense cores of air showers landing close to the monitor. Rough calculations using the event rate (approximately 5 per day) and NKG formula $[11,12]$ indicate that the typical shower results from a $500 \mathrm{TeV}$ primary landing within $\sim 5$ meters of the monitor. In this display, the half circles at the top indicate the position of each Unit. Time runs vertically, early at the bottom and late at the top. The blue lines are at one millisecond intervals and the vertical line separates the bares from the monitors. Each hit is represented by a closed circle, with an area proportional to the amplitude of the signal. Most of the black dots are characteristic of isolated neutron detections with the larger ones resulting from pile-up. Two discriminators are used in the electronics. Simulations show that the signal from a Unit is often quite complex. The typical red dot results from a timing inconsistency between the firing of the discriminators. The semi-periodic spacing of the hits is due to the finite deadtime (approximately 90 microseconds) of the hit measurement system. We are now testing a prototype system that will return a time profile of these complex signals for offline analysis. As normally envisioned, multiplicity would be the count of hits in each Unit, with the Units considered independent. Considering all the hits as an event clearly gives a much better picture of what is actually happening in the monitor.

Air shower catches can show significant structure. The event on the left is rather uniform across the monitor, the center shows a gradient, while the event on the right has a more complex structure. In most large events the bares show signals coincident with the start of the event, but often the signal is not consistent with a neutron detection. Probably they are due to the electromagnetic component of the shower. It is not clear whether the response of the leaded detectors is due to hadrons in the shower or excitation of the lead by the electromagnetic component. Most of the actual detections are neutrons. While new insights with respect to air showers are unlikely, we plan to use these events better to understand the response of the monitor. Our primary concern at this time is identification of interactions that result from primaries in the energy range relevant to modulation. In doing this we are being guided by the results of simulations.

\section{Simulations}

Our simulations show that incident particles in the energy range one to a few hundred $\mathrm{GeV}$ produce quite characteristic signals in the monitor, very unlike the signals in the top row of Figure 2. Our main effort at present is to characterize these events so as to identify them in the data stream and deduce the energy spectrum of the incident particles. Relating this to the spectrum of the primary cosmic rays will be the next stage in our analysis.

There are several steps in our simulation. In each case there is a single incident particle of a specified energy. First, all deposits of energy, with the time of the deposit, in each Unit are recorded using FLUKA [13, 14]. Second, the individual energy deposits are processed by a simulation of the pre-amplifier attached to the anode of the BF3 proportional counter. Third, the simulated 
waveforms are processed through a detailed simulation of the logic of the DAQ to produce a series of hits recorded in individual HitSpools. Finally, the HitSpools are processed by a modified version of the main DAQ program to generate catches using the same logic used to take the actual data.

Examples of simulated events are shown in Figure 2, lower row. Each event resulted from a single, $100 \mathrm{GeV}$ neutron incident as indicated in Figure 1. In the simulation, hits in a minimum of three Units were required to produce a catch. Approximately $30 \%$ of $100 \mathrm{GeV}$ neutrons produced a catch at this level. Events in the figure were selected to have rather large total numbers of hits but are otherwise typical. In each of the three events shown, the first hit was recorded less than 50 microseconds after the incident neutron entered the monitor. These rapid onsets, all due to neutron detections, were somewhat surprising given the typical millisecond time scale of the neutron moderation and interaction exhibited by the remainder of the event. Independent experimental studies with incident protons detected in a plastic scintillator [15] confirm the rapid onset.

Two things are immediately obvious from the simulations. First, the time history of multiple Units simultaneously is much richer in information about the interaction than a count of the multiplicity in them independently. A typical analysis of NM signals would not recognize these as due to a single atmospheric secondary particle. Second, the structure is clearly distinct from the air shower interactions shown in top row of Figure 2. Indeed, the events have a systematic structure. The Unit most directly in the path of the signal has the largest number of hits, while the neutrons spread out in smaller numbers at later times to the adjacent Units. This is consistent with a diffusion-absorption picture of the neutron propagation [15].

While there are many event to event exceptions, hits are typically confined to four or fewer Units with most of the hits in one or two Units. This remains true even when the incident particle enters on the "wings" of the lead producer and/or at non-vertical incident angles. In these cases the hits tend to be more evenly distributed between two Units but the total number of hits and the spread of the distribution stay relatively constant. Finally, the maximum number of hits from incident particles increases nearly linearly with particle energy. Unfortunately, high energy incident particles produce many events that are indistinguishable from those initiated by lower energy particles.

This is illustrated in Figure 3 where the horizontal axis is the number of Units showing hits while the vertical axis is the total number of hits, summed over all Units. The upper left panel is for a $1 \mathrm{GeV}$ (monoenergetic) pencil beam incident as in Figure 1 and the upper center is for $100 \mathrm{GeV}$. The upper right panel shows a simulation of an $E^{-1.0}$ spectrum of neutrons from 1 to $100 \mathrm{GeV}$, distributed over the monitor with incident angles from 0 to 45 degrees.

\section{Data and Analysis}

Figure 3 (lower left) shows the same simulation weighted as an $E^{-2.5}$ spectrum. The lower center panel shows approximately two days of data taken at PSNM using the catch criterion (3/3: three or more Units, three or more total hits). This condition generates approximately 300 megabytes of data per day, so typically a more restrictive requirement, such as $3 / 8$, is used. This distribution bears some resemblance to the simulation, but has a clear "tail" to the right where more Units generate similar numbers of total hits. Some of these catches are from dense cores of air showers, but inspection reveals many that look like they originate from only a few secondary particles from an air shower. 

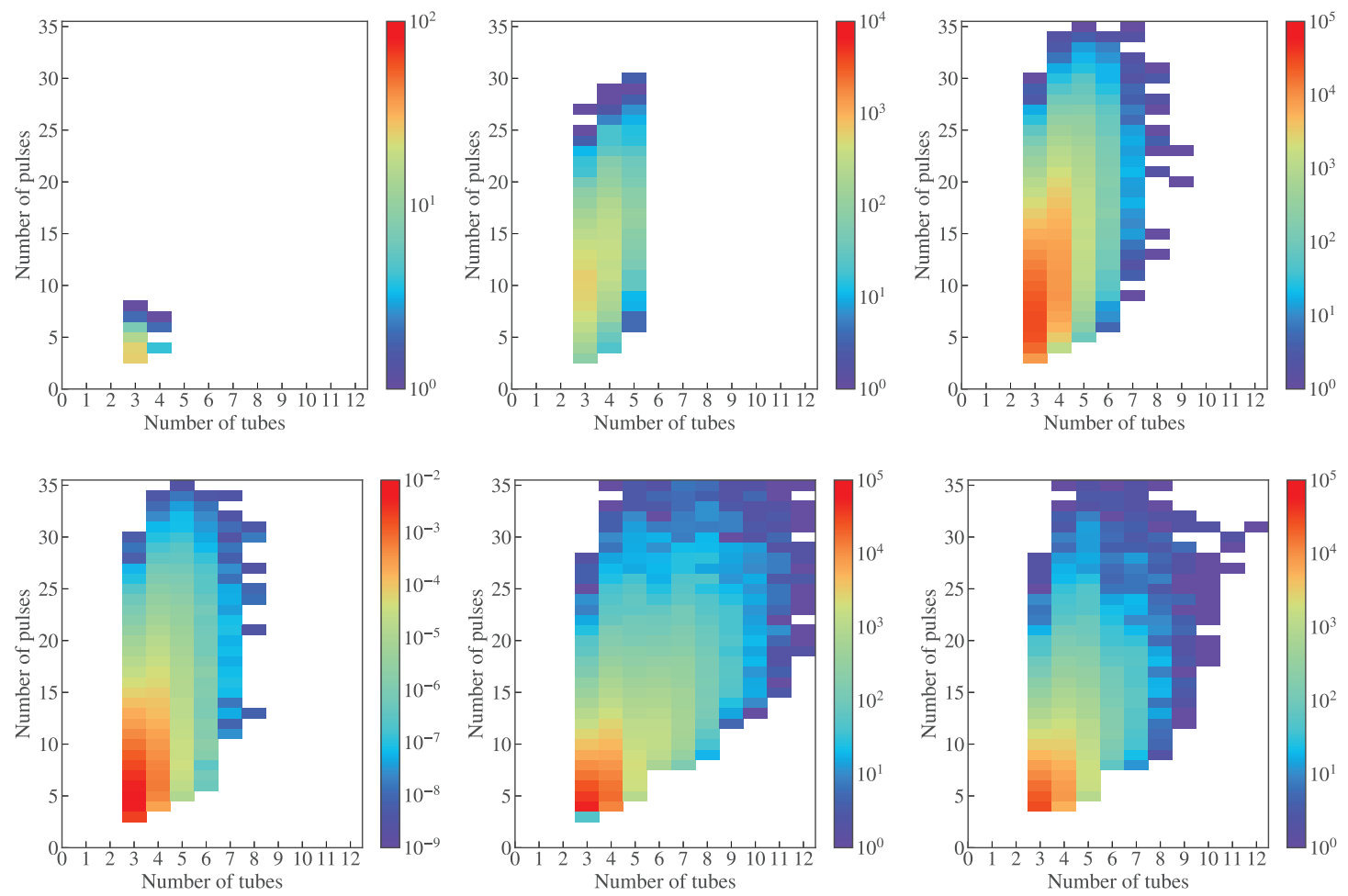

Figure 3: Response of PSNM to incident particles. Top left two panels: Simulated 1 and $100 \mathrm{GeV}$, pencil beam, normal incidence. Top right: Simulated 1 to $100 \mathrm{GeV} E^{-1.0}$ spectrum, uniform illumination at variable zenith angles. Bottom left: The 1 to $100 \mathrm{GeV}$ spectrum re-weighted to $E^{-2.5}$. Bottom center: Approximately two days of data from PSNM. Bottom right: Compact (as defined in the text) subset of actual data.

Our present approach is to concentrate on interactions that are due to single incident particles. The characteristic structure suggests that some type of "machine learning" approach can be constructed, and even applied in real time to reduce the data volume. For now, we are exploring more heuristic cuts, such as that shown in the lower right panel of Figure 3, which illustrates compact catches, defined as having a single group of contiguous Units with hits, and excluding any catch containing a hit in either end Unit of the monitor.

Further analysis will require treating the distribution as a whole to remove background and appropriate fitting to extract the spectrum of the particles incident on the monitor. At this point we explore the intuitive notion that the background is less important for events where fewer total Units are involved. Such an analysis, constructed from the columns in the lower right panel of Figure 3, is shown in Figure 4. In the upper left panel the column of total hits for exactly three Units hit is displayed as a histogram, with the data as the red points. The histogram is truncated at seven total hits because the simulation (at this time) has only been done between 1 and $100 \mathrm{GeV}$. Guided by Figure 3 incident particles below $1 \mathrm{GeV}$ will contribute minimally to this histogram. The weighting of the simulated data is then adjusted as a power law to produce the best fit (minimum chi-square) indicated by the blue line. We can also compute the mean position of the hit cluster from data and simulation as shown in the top center panel. In both cases the data follow the simulation within errors. 

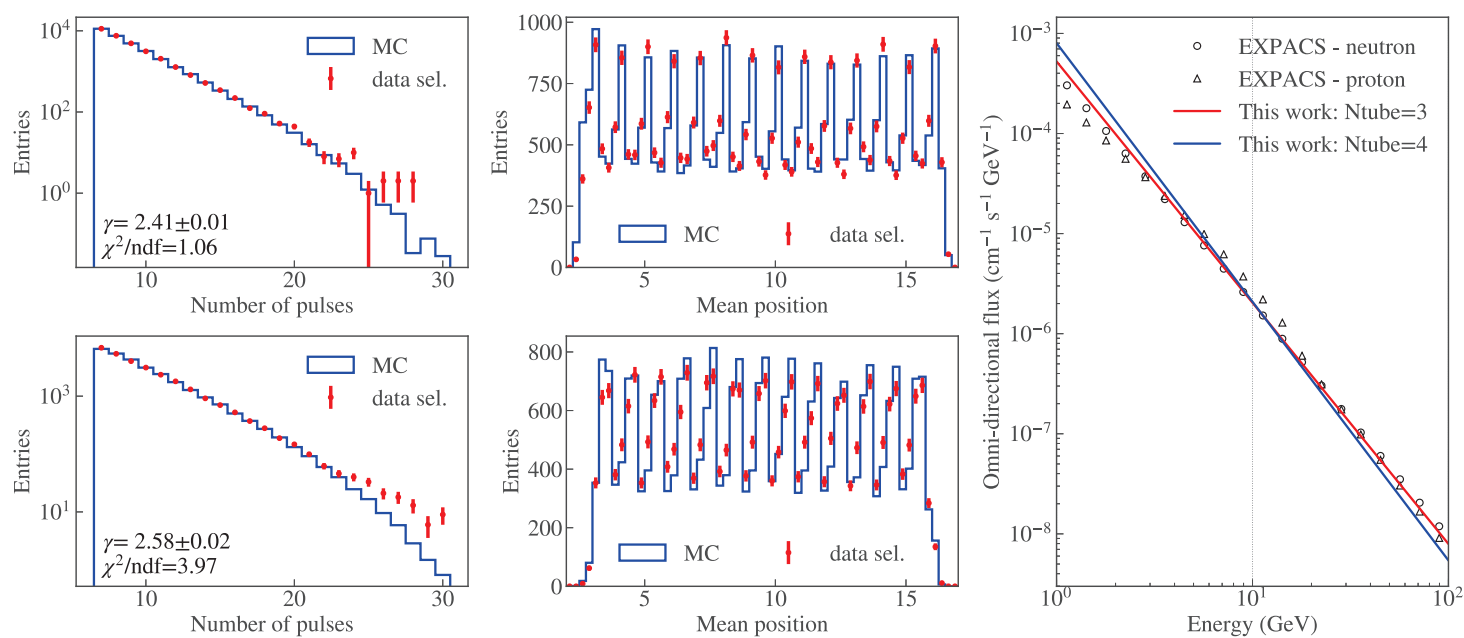

Figure 4: Results of fitting compact data further restricted as discussed in the text.

While this may resemble a conventional multiplicity deconvolution it is actually much more constrained, since the events are selected based on groups of Units and the multiplicity is calculated from the total number of hits in the group. The lower left and center panels illustrate the calculation for the column containing four and only four Units. The fits are still reasonable, but clearly not as good as the three Unit case.

The expected spectrum of incident particles calculated by the EXPACS 410 program [16-18] for the time and location of the data is shown in the right panel of Figure 4 as black symbols. EXPACS calculates both protons and neutrons, whereas our simulation to date only considers neutrons. The best fit spectra from the left panels (arbitrarily normalized at $10 \mathrm{GeV}$ ) are shown as red (for 3 Units) and blue (for 4 Units) lines. For such a simple analysis we find the agreement remarkable, with some indication of better agreement for the case assumed to have less background.

\section{Discussion and Conclusions}

By examining the details of multi particle detections at PSNM we are able to produce estimates of the spectrum of secondary particles incident on the monitor. With further modeling and simulation we should be able to relate these spectra to the spectra of primary cosmic rays above the $17 \mathrm{GV}$ cutoff at PSNM with sufficient statistics to track the solar modulation of these spectra. A key part of this effort will be understanding the inevitable dependence of the observed secondary spectra on barometric pressure and possibly other aspects of atmospheric structure. We are now taking data at PSNM with the objective of tracking the evolution of the spectrum through the upcoming solar maximum and magnetic polarity reversal. We would like to thank Serap Tilav, Dennis Soldin, Dave Seckel and Tom Gaisser for many helpful discussions. Supported in part by grant RTA6280002 from Thailand Science Research and Innovation and United States NSF Award 1341312.

\section{References}

[1] Moraal, H., M.S. Potgieter \& P.H. Stoker, Neutron Monitor Latitude Survey of Cosmic Ray 
Intensity During the 1986/1987 Solar Minimum, J. Geophys. Res. 94, 1459-1464, 1989

[2] Nuntiyakul, W., et al., Latitude Survey Investigation of Galactic Cosmic Ray Solar Modulation During 1994 - 2007, Astrophys. J. 795:11, doi:10.1088/0004-637X/795/1/11, 2014

[3] Mangeard, P.-S., et al., Distinct Pattern of Solar Modulation of Galactic Cosmic Rays above a High Geomagnetic Cutoff Rigidity, Astrophys. J. 858:43, doi:10.3847/1538-4357/aabd3c, 2018

[4] Jokipii, J.R., E.H. Levy \& W.B. Hubbard, Effects of Particle Drift on Cosmic-Ray Transport. I. General Properties, Application to Solar Modulation, Astrophys. J. 213,861-868, 1977

[5] Bieber, J.W., P.A. Evenson \& W.H. Matthaeus, The Nuts and Bolts of Cosmic Ray Modulation, Proc. 20th ICRC (Moscow) 3, 175-179, 1987

[6] Dyring, E. \& B. Sporre, Latitude effect of neutron multiplicity as detected by a shipborne neutron monitor, Ark. Geofys. 5, 67, 1966

[7] Ruffolo, D., et al., Monitoring short-term cosmic-ray spectral variations using neutron monitor time-delay measurements, $A p J \mathbf{8 1 7}, 38,2016$

[8] Banglieng, C., et al., Tracking Cosmic-Ray Spectral Variation during 20072018 Using Neutron Monitor Time-delay Measurements, Astrophys. J. 890, 21, 2020

[9] Sáiz, A., et al., Measurement of cross-counter leader fractions in an 18NM64: Detecting single and multiple atmospheric secondaries, Proc. 35th ICRC (Busan) PoS(ICRC2017)047, 2017

[10] Sáiz, A., et al., Detecting single and multiple atmospheric secondaries in an 18NM64, Proc. 36th ICRC (Madison) PoS(ICRC2019)1145, 2019

[11] Antoni, T., et al., Electron, muon, and hadron lateral distributions measured in air showers by the KASCADE experiment, Astroparticle Physics 14, 245-260, 2001

[12] Antoni, T., et al., The cosmic-ray experiment KASCADE, NIM A 513, 490 510, 2003

[13] Battistoni, et al., Overview of the FLUKA code, Annals of Nuclear Energy 82, 10-18, 2015.

[14] Bohlen, T.T., et al., The FLUKA Code: Developments and Challenges for High Energy and Medical Applications, Nuclear Data Sheets 120, 211-214, 2014

[15] Chaiwongkhot K., et al., Measurement and simulation of the neutron propagation time distribution inside a neutron monitor, Astroparticle Physics 132, 102617, 2021

[16] Sato, T., Analytical model for estimating terrestrial cosmic ray fluxes nearly anytime and anywhere in the world: Extension of PARMA/EXPACS, PLOS ONE 10(12) e0144679, 2015

[17] Sato, T., Analytical Model for Estimating the Zenith Angle Dependence of Terrestrial Cosmic Ray Fluxes, PLOS ONE11(8) e0160390, 2016

[18] https://phits.jaea.go.jp/expacs/ 